YITP-97-4, February 1997

\title{
Level Statistics of Near-Yrast States in Rapidly Rotating Nuclei
}

\author{
M. Matsuo, T. Døssing ${ }^{a}$, E. Vigezzi ${ }^{b}$, and S. Åberg ${ }^{c}$ \\ Yukawa Institute for Theoretical Physics, Kyoto University, Kyoto 606-01, Japan \\ a Niels Bohr Institute, University of Copenhagen, Copenhagen Ø, Denmark \\ ${ }^{b}$ INFN sez. Milano, University of Milan, Milan, Italy \\ ${ }^{c}$ Department of Mathematical Physics, Lund Institute of Technology, Lund, Sweden
}

\begin{abstract}
The nearest neighbour level spacing distribution and the $\Delta_{3}$ statistics of level fluctuations associated with very high spin states $(I \gtrsim 30)$ in rare-earth deformed nuclei are analysed by means of a cranked shell model. The many particle-many hole configurations created in the rotating Nilsson potential are mixed by the surface-delta two-body residual interaction. The levels in the near-yrast region show a Poisson-like level spacing distribution. As the intrinsic excitation energy $U$ increases, the level statistics shows a gradual transition from order to chaos, reaching at $U \gtrsim 2 \mathrm{MeV}$ the Wigner distribution typical of the Gaussian orthogonal ensemble of random matrices. This transition is caused by the residual two-body interaction. On the other hand, the level spacings between the yrast and the first excited state show a peculiar behaviour, displaying a Wigner-like distribution instead of the Poisson-like distribution seen for the other near-yrast rotational states. The lowest spacings reflect the properties of the single-particle orbits in the mean-field, and are only weakly affected by the residual two-body interaction.
\end{abstract}

PACS: 21.10.Re, 21.60.Ev, 23.20.Lv, 24.60.-k, 24.60.Ky, 24.60.Lz

Keywords: high spin states, level statistics, chaos, cranked Nilsson potential, surface delta interaction

\section{Introduction}

The statistical fluctuations of the energy levels and the transition strengths measured in highly excited nuclei with excitation energy above the neutron threshold (several MeV) are well described by the random matrix theory [1, 2, 3]. For example, the nearest-neighbour level spacing distribution (NND) and the spectral rigidity (or $\Delta_{3}$ statistics) of the neutron resonance states follow the behaviour predicted by the random matrix theory for the Gaussian orthogonal ensemble (GOE) 11, 国. This seems to indicate that such excited nuclei, at least over a time scale associated with the observed energy interval, are an example of a chaotic quantal system, in the sense that GOE fluctuations generally characterize quantum systems which are chaotic in the classical limit [5, 6.

The fluctuation properties at lower excitation energy are less well understood, although several extensive analysis of low-lying levels as well as of near-yrast high spin levels have been reported recently $17,8,9,10,11,12,13$. Although the low lying and low spin levels generally show level spacing distributions which are intermediate between chaos (the GOE or Wigner limit) and order (the Poisson limit), one observes some systematic behaviour with respect to the mass-number and the angular momentum [8]. In particular, it is remarkable that the NND in heavy deformed nuclei is the closest to the Poisson distribution, not only for the low-lying, low spin levels [8], but also for the high spin rotational levels lying near the yrast line [11, 12, 13. This suggests that both the Poisson 
and the GOE fluctuations coexist in rotating nuclei and that one should expect a transition from order to chaos with increasing intrinsic excitation energy $U$ (the relative excitation energy measured from the yrast line at given spin). In the present paper, we examine theoretically the level statistics of high spin states in rapidly rotating nuclei as a function of intrinsic excitation energy $U$. In particular, we investigate in detail the level statistics associated with the near-yrast states which may become accessible in future experiments. We limit ourselves to the very high spin region with $I \gtrsim 30$, where static pairing is generally quenched or even vanishes, because our model is not adequate to deal with strong pairing correlations. Although this makes it difficult to make a direct comparison with present experimental data, there are good reasons to expect that much more experimental information will be available in the near future.

The high spin states near the yrast line in well deformed nuclei form rotational band structures, as evidenced by experiments. These rotational band states are usually well described by the cranked mean-field models [14, 15, 16] in which the collective rotation is represented by uniform rotation along the axis of the largest moment of inertia (axis perpendicular to the elongated direction). The intrinsic structure of a rotating nucleus is described in terms of the mean-field potential adding the cranking term caused by the uniform rotation. Most observed rotational bands are based on intrinsic configurations with a few excited quasiparticles (or particles and holes) defined in the cranked meanfield Hamiltonian. However, as the intrinsic excitation energy $U$ increases at a given spin, intrinsic configurations with many particles and holes $(n \mathrm{p}-n \mathrm{~h})$ will show up and become progressively dominant. Accordingly, the level density increases significantly, reaching a value around $\sim 10^{2} / \mathrm{MeV}$ at intrinsic excitation energy $U \sim 1 \mathrm{MeV}$ above yrast line in rare earth nuclei. One then expects that the residual two-body interaction begins to play an important role, mixing the $n \mathrm{p}-n \mathrm{~h}$ configurations, because the size of its matrix elements $(\sim 10 \mathrm{keV})$ is of the same order as the mean level spacing. It is also to be noticed that the phenomenon of the rotational damping [17], which sets in at around $U \sim 0.8 \mathrm{MeV}$ above yrast [18, is an important signature of the configuration mixing caused by the residual two-body interaction. The fluctuations of the energy levels will be sensitive to the configuration mixing among the $n \mathrm{p}-n \mathrm{~h}$ configurations. If the configuration mixing were absent, intrinsic excitations would be specified uniquely by the excited particles and holes. In such a situation, the level fluctuations may follow the Poisson distribution. On the other hand, once the residual twobody interaction is switched on, the $n \mathrm{p}-n \mathrm{~h}$ configurations interact with each other. If the residual interaction is so strong that many $n \mathrm{p}-n \mathrm{~h}$ configurations are admixed with complicated amplitudes, one expects that the level fluctuations obey the theory of random matrices. It is therefore important, in studying the level fluctuations as a function of intrinsic excitation energy, to take configuration mixing explicitly into account. We adopt a shell model approach, making use of a reasonable residual two-body interaction on top of a cranked mean field [19, 20. Previous work with the cranking model [19] has already discussed some general features of the order to chaos transition although it used a schematic residual interaction represented by a constant with random sign. We have recently shown that the cranked Nilsson model combined with the surface-delta interaction (SDI) [21, 22] can reproduce the overall features of rotational damping found in experiment [20, 23, 24]. In the present paper we adopt the same model, studying the excited levels lying up to about $2 \mathrm{MeV}$ above yrast line. We study in particular detail the states close to yrast, which are likely to be observed in near future experiments.

Statistical analyses of high spin levels in deformed nuclei on the basis of the interacting boson model [25], the interacting boson fermion model [26], and the particle-rotor model [27] have also been reported. These models, however, take into account only limited degrees of freedom (sd collective bosons or high- $j$ nucleons) of the intrinsic excitations in deformed rotating nuclei.

\section{Formulation}

\subsection{The model}

We start with the cranked Nilsson single-particle Hamiltonian

$$
h_{\text {crank }}=h_{N i l s s o n}-\omega j_{x}
$$


in order to define the single-particle basis in a rotating deformed nucleus. Here the quadrupole and hexadecapole deformations are considered. We do not include the static pairing potential in the mean-field. This may be justified for the high spin region $(I \gtrsim 30)$ which we are mostly concerned with, since the pairing gap is usually reduced, or even vanishes, due to the rotational perturbation (Mottelson-Valatin effect) [28, 29, 30]. The eigen-solutions of the cranked Nilsson single-particle Hamitonian define an adiabatic basis as a function of the rotational frequency $\omega$. However, since the adiabatic orbits sometimes accompany avoided crossings between orbits which cause abrupt change of the basis wave functions against small change in $\omega$, we instead use a diabatic single-particle basis, which is constructed by removing small interactions causing the repulsions at the avoided crossings. Putting $N$ neutrons and $Z$ protons in the diabatic single-particle basis, shell model many-body configurations (labeled by $\mu$ ) are generated:

$$
|\mu(I)\rangle=\prod_{\text {occupied } i \text { in } \mu} a_{i}^{\dagger}|-\rangle .
$$

In Eq.(2) $a_{i}^{\dagger}$ denotes the nucleon creation operator for an occupied diabatic single-particle orbit $i$, which is defined at an average rotational frequency $\omega_{I}$ corresponding to the given angular momentum $I$. We include all the single-particle orbits within an interval of $3.0 \mathrm{MeV}$ below and above the Fermi surface. The shell model basis $\{|\mu(I)\rangle\}$ includes the configuration in which the single-particle orbits up to the Fermi surface are fully occupied, as well as all possible $n \mathrm{p}-n \mathrm{~h}$ configurations with respect to the fully occupied one.

The energy of a shell model configuration $|\mu(I)\rangle$ is given, following the standard cranked NilssonStrutinsky prescription, by

$$
E_{\mu}(I)=E_{\mu}^{N i l s}(I)-E^{\text {smooth }}(I)+E^{R L D}(I)
$$

where $E_{\mu}^{\text {Nils }}(I)=E_{\mu}^{\prime}(\omega)+\omega J_{x, \mu}(\omega)$ with the angular momentum constraint $J_{x, \mu}(\omega)=I$ on the rotational frequency $\omega$. Here $E_{\mu}^{\prime}(\omega)=\sum_{i \in \mu} e_{i}^{\prime}(\omega)$ and $J_{x, \mu}(\omega)=\sum_{i \in \mu} j_{x, i}(\omega)$ are the total routhian and the expectation value of the angular momentum $J_{x}$ of the shell model basis $\mu$, respectively. Since we use the diabatic single-particle basis which depends only weakly on the rotational frequency, the energy expression can be accurately approximated locally by

$$
E_{\mu}^{N i l s}(I)=E_{\mu}^{\prime}\left(\omega_{I}\right)+\omega_{I} I+\frac{\left(I-J_{x, \mu}\left(\omega_{I}\right)\right)^{2}}{2 J_{\mu}^{(2)}}
$$

referring to the average rotational frequency $\omega_{I}$. Here $J_{\mu}^{(2)}$ is the dynamical moment of inertia of the configuration. The deviation $\left|J_{x, \mu}\left(\omega_{I}\right)-I\right|$ in the angular momentum expectation value is less than 5 at spin $I=50$ for most configurations in the present calculation. Although the Strutinsky smoothed energy $E^{\text {smooth }}(I)$ and the rotating liquid drop energy $E^{R L D}(I)$ correct the absolute excitation energy, they do not affect the level statistics discussed in the present paper.

We then introduce a two-body force, mixing the shell-model configurations. We adopt the surface delta interaction (SDI) 21]

$$
v(1,2)^{\text {angle }}=-4 \pi V_{0} \sum_{L M} Y_{L M}^{*}\left(\theta_{t, 1} \phi_{t, 1}\right) Y_{L M}\left(\theta_{t, 2} \phi_{t, 2}\right)
$$

where $\left(\theta_{t} \phi_{t}\right)$ is the angle variable in the stretched coordinates. The strength parameter $V_{0}$ includes the radial matrix elements and we use the strength $V_{0}=27.5 / A \mathrm{MeV}$ given by Ref.[22], which is the same value used for the study of rotational damping in ${ }^{168} \mathrm{Yb}$ [20, 23]. The shell model Hamiltonian is given by

$$
H(I)_{\mu \mu^{\prime}}=E_{\mu}(I) \delta_{\mu \mu^{\prime}}+V(I)_{\mu \mu^{\prime}}
$$

where $V(I)_{\mu \mu^{\prime}}$ denotes the matrix elements of the residual two-body interaction of SDI. The Hamiltonian is diagonalized to obtain energy eigenstates

$$
|\alpha(I)\rangle=\sum_{\mu} X_{\mu}^{\alpha}(I)|\mu(I)\rangle
$$




\begin{tabular}{|c|cc|}
\hline & $\epsilon_{2}$ & $\epsilon_{4}$ \\
\hline${ }^{160,161} \mathrm{Dy}{ }^{161,162} \mathrm{Ho}$ & 0.248 & -0.016 \\
${ }^{162,163} \mathrm{Dy}{ }^{163,165} \mathrm{Dy}{ }^{165,166} \mathrm{Ho}$ & 0.261 & -0.007 \\
${ }^{162,163} \mathrm{Er}{ }^{163,164} \mathrm{Tm}$ & 0.267 & 0.003 \\
${ }^{164,165} \mathrm{Er}{ }^{165,166} \mathrm{Tm}$ & 0.255 & -0.009 \\
${ }^{166,167} \mathrm{Er}{ }^{167,168} \mathrm{Tm}$ & 0.267 & 0.001 \\
${ }^{166,167} \mathrm{Yb}{ }^{167,168} \mathrm{Lu}$ & 0.246 & 0.004 \\
${ }^{170,169} \mathrm{Yb}{ }^{169,170} \mathrm{Lu}$ & 0.255 & 0.014 \\
${ }^{172,173} \mathrm{Yb}{ }^{171,172} \mathrm{Lu}$ & 0.265 & 0.025 \\
\end{tabular}

Table 1: The quadrupole and hexadecapole deformation parameters $\epsilon_{2}$ and $\epsilon_{4}$ used in the present calculations

which are admixtures of the basis configurations $\{|\mu(I)\rangle\}$ as well as their energy levels $\left\{E_{\alpha}(I)\right\}$. The diagonalization is done separately for each $I^{\pi}$, truncating the basis by including the lowest $1000|\mu\rangle$ basis states. The resulting lowest 300 states (covering the region up to $U \sim 2.4 \mathrm{MeV}$ ) are rather stable against the truncation of the basis. For further details of the model, we refer to Ref. [20].

In the present paper, we focus on the rare-earth nuclei, and in particular we consider 40 nuclei in the $A=160-174$ region, listed in Table 1, for which deformed prolate shape stable up to very high spins is suggested by potential energy surface calculations [31, 32]. We adopt the equilibrium deformation parameters taken from Ref.[33], as given in Table 1, which are similar to those calculated in Ref.[31]. In order to make a statistically meaningful analysis, we collect the spacings taken from a certain spin interval in all the 40 nuclei, and we will not discuss the dependence on individual nuclei, spins, and parities.

In the following, we use the parity and the signature quantum number $(\pi, \alpha)$ to classify the energy levels of the total system. The signature $\alpha$ is related to the total spin $I$ through the relation $I=I_{0}+\alpha$ with $\alpha=0,1$ for even- $A$ system, and $\alpha= \pm 1 / 2$ for odd- $A$. We sometimes use the even integer spin $I_{0}$ and the signature $\alpha$ in place of the "true" spin $I$ when we specify spin intervals.

\section{$2.2 \quad$ Level statistics}

In order to perform the statistical analysis of the energy level fluctuations, one must take into account the fact that the level density and hence the level spacing are strongly dependent on the intrinsic excitation energy $U$. In this situation, it is necessary to separate local level fluctuation from the overall excitation energy dependence of the level spacings. For that purpose, we adopt the unfolding procedure [34, 5] in a particular form which follows Shriner et.al. [8]. The unfolding procedure measures the local level fluctuations with respect to a smooth average level density. We assume that the average level density is represented by the constant temperature formula [35]

$$
\bar{\rho}(E)=\frac{1}{T} \exp \left(\frac{E-E_{0}}{T}\right)
$$

for each spectrum at a given $I^{\pi}$. To determine the parameters in the formula, we make a fit to the staircase function which represents the cumulative number of levels below energy $E$,

$$
N(E)=\int_{-\infty}^{E} \rho\left(E^{\prime}\right) d E^{\prime}=\sum_{\alpha} \theta\left(E-E_{\alpha}\right)
$$

with a smooth function corresponding to the average level density $\bar{\rho}(E)$,

$$
\bar{N}(E)=\int_{E_{0}}^{E} \bar{\rho}\left(E^{\prime}\right) d E^{\prime}+N_{0}=\exp \left(\frac{E-E_{0}}{T}\right)-1+N_{0}
$$


by minimizing the quantity

$$
G\left(T, E_{0}, N_{0}\right)=\int_{E_{\min }}^{E_{\max }}(N(E)-\bar{N}(E))^{2} d E
$$

with respect to the parameters $T, E_{0}$ and $N_{0}$ for each spectrum at a given $I^{\pi}$. Here the energy boundaries $E_{\min }$ and $E_{\max }$ are the energies of the lowest and the 300-th levels in each spectrum. When we discuss the level statistics for the lowest 20 levels, however, we obtain a better fit including only the lowest 30 levels. The unfolded spectra $\left\{x_{\alpha} ; \alpha=1,2, \ldots\right\}$ are then derived for each $I^{\pi}$ by the transformation

$$
x_{\alpha}=\bar{N}\left(E_{\alpha}\right) .
$$

The unfolded spectra have a constant average density $\bar{\rho}_{x}(x)=1$ provided that the constant temperature formula fits well the average level density.

In order to analyze the level fluctuations, we calculate the nearest neighbour level spacing distribution (NND) which is also often used in the experimental analysis. We calculate the distribution $P(s)$ for the unfolded spectra, where $s=x_{\alpha+1}-x_{\alpha}$ is the spacing between the neighbouring levels with same $I^{\pi}$. By the unfolding procedure, the spacings are normalized as $\langle s\rangle=1$. The distribution is represented as a histogram.

The NND is calculated for various ensembles of level spacings which are taken from different intervals in excitation energy. The obtained distribution is fitted with the Brody distribution [1]

$$
P_{w}(s)=(1+w) \alpha s^{w} \exp \left(-\alpha s^{1+w}\right), \quad \alpha=\left(\Gamma\left(\frac{2+w}{1+w}\right)\right)^{1+w},
$$

parametrized by the Brody parameter $w$. This family of distributions is convenient because the Brody parameter $w=1$ produces the Wigner distribution while the value $w=0$ corresponds to the Poisson distribution. (Note that the theory of GOE random matrices leads to $w=0.953$ [西, which is not distinguishable from the Wigner limit in the present analysis). The value of $w$ is determined minimizing the quantity

$$
S(w)=\sum_{i}\left(\frac{P(i)-P_{w}(i)}{\sigma(i)}\right)^{2}
$$

where $P(i)=N(i) / N$ is the probability in the $i$-th bin $\left[s_{i}, s_{i}+\Delta s\right]$ of the calculated NND $(N(i)$ being the number of spacings in bin $i$ out of the total spacings of $N) . P_{w}(i)=\int_{s_{i}}^{s_{i}+\Delta s} P_{w}\left(s^{\prime}\right) d s^{\prime}$ is the corresponding probability in the Brody distribution. The statistical error is estimated as $\sigma(i)=\sqrt{N(i)} / N$ for $N(i)>0$ and $\sigma(i)=1.15 / N$ for $N(i)=0$ by assuming the multinomial distributions.

We also calculate the ensemble average of the $\Delta_{3}$ statistics [36, 1]

$$
\bar{\Delta}_{3}(L)=\left\langle\frac{1}{L} \min _{A, B} \int_{x}^{x+L}\left[N_{x}\left(x^{\prime}\right)-A x^{\prime}-B\right]^{2} d x^{\prime}\right\rangle
$$

or the spectral rigidity. Here $N_{x}(x)=\sum_{\alpha} \theta\left(x-x_{\alpha}\right)$ is the staircase function for the unfolded spectra and the average $\langle\ldots\rangle$ is calculated over spectra in a given ensemble and intervals $[x, x+L]$, $[x+L / 2, x+3 L / 2], \ldots$ in a spectrum [5]. For the Poisson distribution of levels,

$$
\bar{\Delta}_{3, \text { Poisson }}(L)=L / 15
$$

while for the GOE distribution,

$$
\bar{\Delta}_{3, \mathrm{GOE}}(L) \approx \frac{1}{\pi^{2}}(\ln L-0.0687)
$$




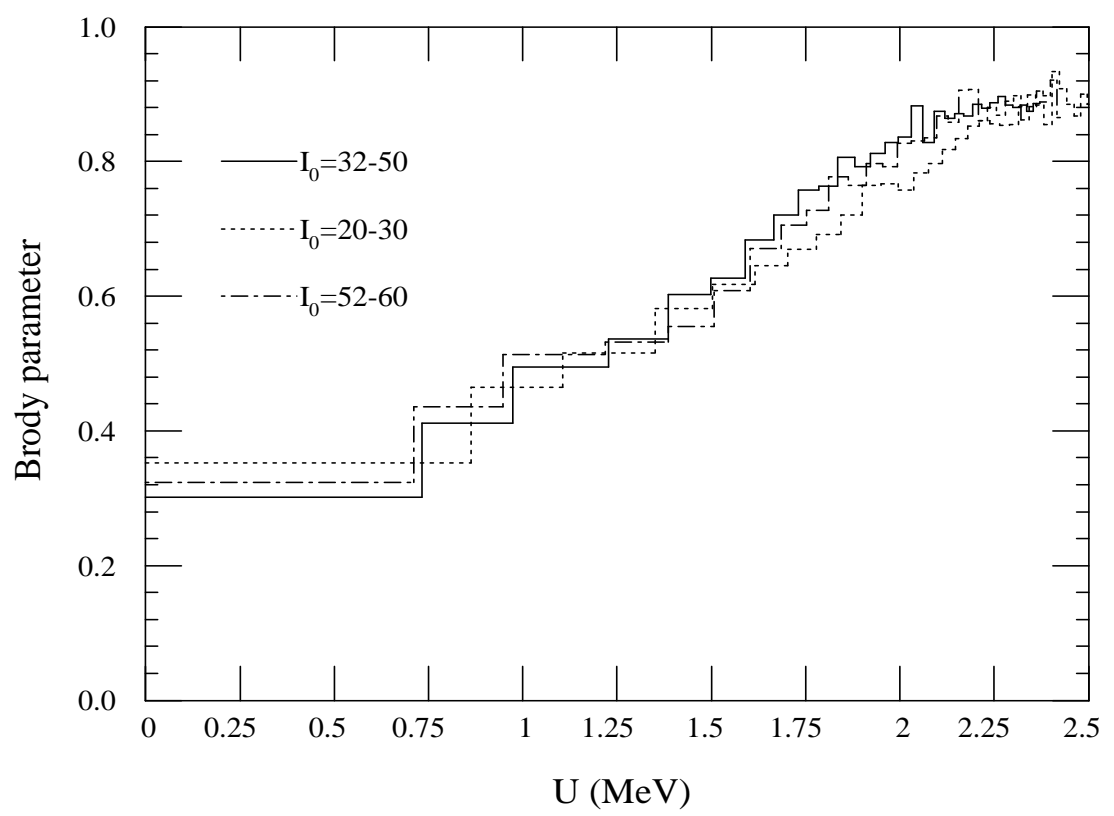

Figure 1: The Brody parameter extracted from the NND for energy bins containing the first to 5 -th, 6-th to 10-th, 11-th to 20-th, 21-st to 30th, and 31-st to 40-th levels, ... 291-st to 300-th of each spectrum. The result is plotted as a function of the intrinsic excitation energy covered by the bins. The solid, dotted, and dot-dashed lines correspond to different spin intervals $I_{0}=$ $32-50,20-30,52-60$ respectively.

\section{Results and Discussion}

\subsection{Order to chaos transition}

We first discuss how the level statistics depends on the intrinsic excitation energy $U$, aiming at extracting the overall dependence on $U$ in a wide interval ranging from $U=0$ (at yrast line) to $U \sim 2 \mathrm{MeV}$. For that purpose, we calculate the NND and $\Delta_{3}$ for the lowest 300 levels in each spectrum, grouping them in bins of levels. The intrinsic excitation energy of the binned levels approximately covers the region up to $U \sim 2.4 \mathrm{MeV}$.

The calculated Brody parameter for the NND for the spin interval $I_{0}=32-50$ is depicted in Fig.1. The Brody parameter increases monotonically with increasing intrinsic excitation energy $U$. The NND for the lowest bin (first to 5 -th levels at each $I^{\pi}$ ), has the Brody parameter $w=$ $0.301 \pm 0.012$. The corresponding NND shown in Fig.2(a) is much closer to the Poisson than to the Wigner distribution, although one can also notice a small deviation from the Poisson distribution. For the levels from 10-th to 40-th, the Brody parameter is about 0.5 , which is midway between the Poisson and the Wigner distributions, as can also be seen from the NND plotted in Fig.2(b). As the intrinsic excitation energy increases further, the NND approaches the Wigner limit; the bin including the levels from the 291-st to 300-th has $w=0.888 \pm 0.012$, which is close to the Wigner limit $w=1.0$ or GOE limit 0.953 (See also the NND shown in Fig.2(c)). These results indicate that the transition from order (Poisson fluctuation of the levels) to chaos (Wigner and GOE fluctuations) takes place gradually increasing the intrinsic excitation energy, until the chaotic limit is nearly achieved at around $U \sim 2 \mathrm{MeV}$ above yrast line. This dependence on excitation energy confirms the results of a previous analysis [23] performed with the same model, but without transforming the energy from the rotating frame to the laboratory frame (the last two terms in Eq.(1) were neglected).

It is interesting to note the implications of the results from the $\Delta_{3}$-statistics (Fig.3). The GOE limit is obtained only for $L$ values up to some value $L_{\max }$, and it is found that $L_{\max }$ increases with increasing excitation energy. For the bin at the highest studied excitation energy (\#251 - \#300, $U \sim 2.3 \mathrm{MeV}$ ), we find that $L_{\max } \sim 6$. This implies that an energy eigenstate in this interval follows 

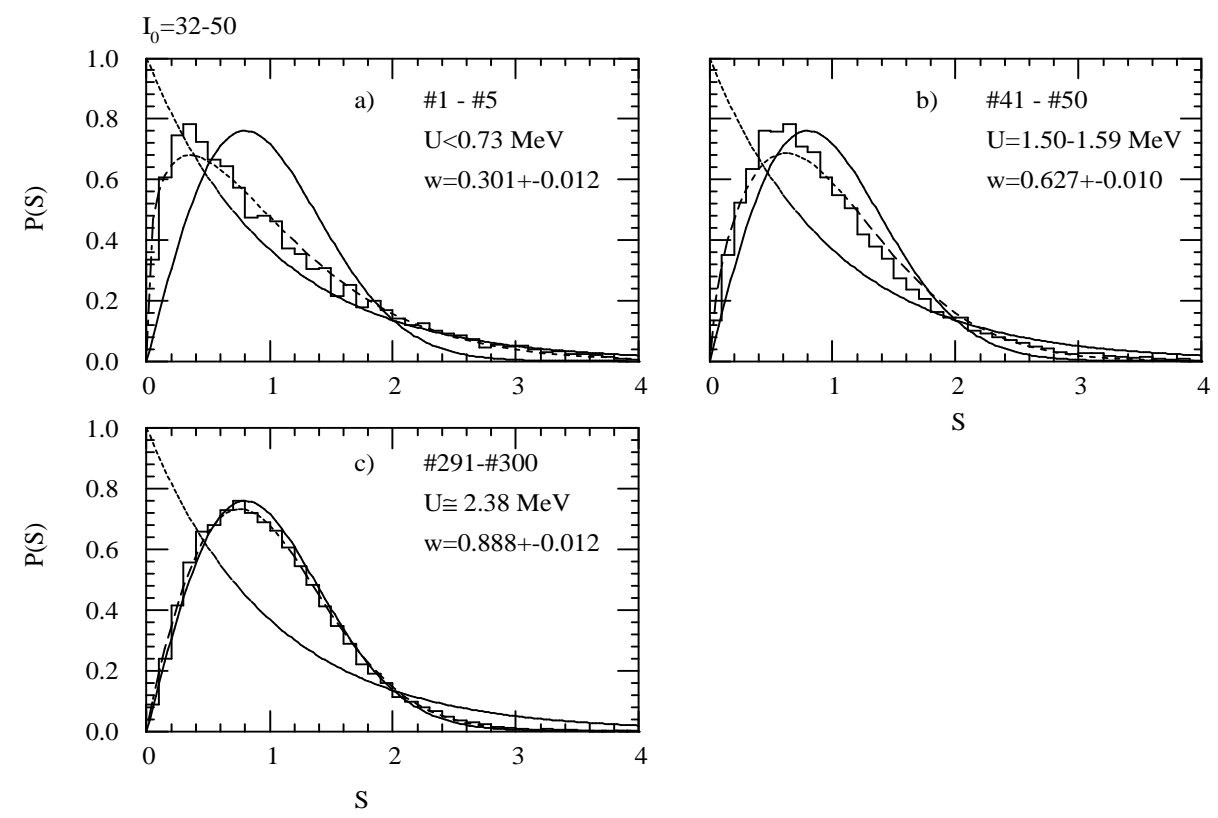

Figure 2: The NND for energy bins containing the first to 5-th, 41-st to 50-th, and 291-st to 300 th levels of each spectrum within spin interval $I_{0}=32-50$.

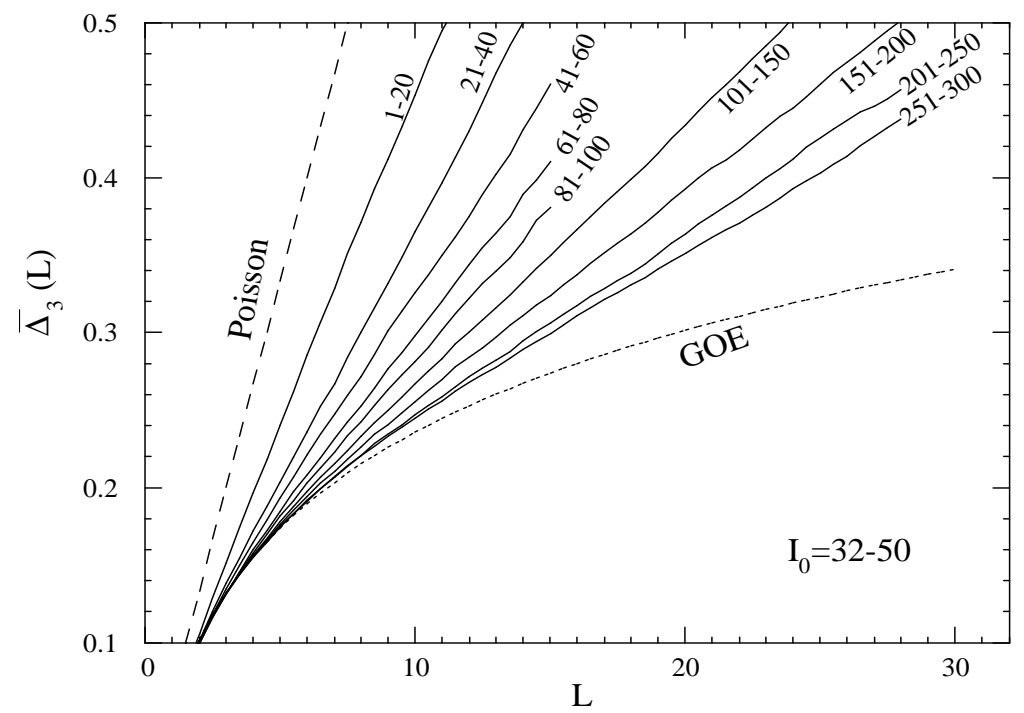

Figure 3: The spectral rigidity $\bar{\Delta}_{3}(L)$ calculated for different energy bins containing 20 or 50 levels in each spectrum with fixed $I^{\pi}$ within the spin interval $I_{0}=32-50$, plotted with solid lines. 
the GOE correlation only with approximately the ten closest lying states. Thus, the GOE-behaviour seen in the NND for the energy levels in this interval (Fig.2(c)) indicates a chaotic behaviour of local nature. The NND and $\Delta_{3}$ carry different types of information for short-range and long-range correlations, as discussed in 37. Since the spreading width $\Gamma_{\mu}$ of $n \mathrm{p}-n \mathrm{~h}$ shell model basis states is finite, $L_{\max }$ could be related to $\rho \Gamma_{\mu}$ ( $\rho$ being the level density) [19, 37 although an estimate based on $L_{\max } \approx 2.5 \rho \Gamma_{\mu}$ which is found in a random matrix model [37] gives a much larger value than the calculated $L_{\max } \sim 6$. Non generic behaviours of $\Delta_{3}$ have also been discussed in connection with the shortest periodic orbits [38] and the Lyapunov exponent [39] in semiclassical analysis, whose relation to the present model is not clear yet.

In Fig.11, we also show the Brody parameters extracted from ensembles of binned levels taken from lower and higher spin intervals with $I_{0}=20-30$ and $I_{0}=52-60$. No significant spin dependence is observed. This is in contrast with the interacting boson fermion model [26] and the particle-rotor model [27], which predict a spin dependence caused by the alignment of the high- $j$ orbitals. Note that, besides the high- $j$ orbitals, we include all the other single-particle orbits near the Fermi surface, which do not necessarily align in the considered spin interval.

It is interesting to compare our results with the previous theoretical analysis by Åberg [19], who used essentially the same model except for the matrix elements of the two-body interaction, which were schematically approximated by a constant with random sign. When the mean square root value of the matrix elements was $15 \mathrm{keV}$, the $\Delta_{3}$ statistics reached the GOE limit in the excitation energy range $U=1.5-2.0 \mathrm{MeV}$ in ${ }^{168} \mathrm{Yb}$, a value lower than in the present model. The difference can be traced back to the statistical properties of the two-body matrix elements. We find that the statistical distribution of the off-diagonal matrix elements of the SDI force follows a distribution strongly peaked at zero matrix element compared with the Gaussian distribution 20, 23], indicating a selectivity in the two-body matrix elements related to the intrinsic nature of the SDI and of the cranked Nilsson single-particle orbits. Because of this selectivity, the onset of chaos in our model takes place at higher excitation energy, although the average mean square root of the off-diagonal matrix elements is about $19 \mathrm{keV}$ in the present calculation.

\subsection{Level statistics in the near-yrast region}

The bin including the lowest 5 levels for each $I^{\pi}$ covers the interval up to $U \sim 0.7 \mathrm{MeV}$ above the yrast line. The calculated levels in this energy region mostly form rotational band structures connected by strong stretched E2 transitions [20]. These levels are probably those which will be resolved in experiments in the near future, while it will be much harder to resolve excited levels lying in the region of rotational damping $(U \gtrsim 0.8 \mathrm{MeV})$. In fact, up to around 10-20 rotational bands are observed in a few rare-earth nuclei [40, 41] (although only a few rotational bands are identified at the highest spin $I \sim 40$ ). In this subsection, we discuss in detail the level statistics associated with these near-yrast states.

For this purpose, we introduce a strict ordering of the spacings according to excitation energy above yrast. The strict ordering $N$ encompasses four spectra having different parity $\pi$ and signature $\alpha$ for a given reference spin $I_{0}$ (even integer), that is those spectra with $I^{\pi}=I_{0}^{ \pm},\left(I_{0}+\right.$ $1)^{ \pm}$for even- $A$ systems, and those with $I^{\pi}=\left(I_{0} \pm 1 / 2\right)^{ \pm}$for odd- $A$ systems. More precisely, we first define a reference energy $E_{r e f}(I)$ by an envelope of the yrast levels, i.e, the $E_{r e f}(I)=$ $\min \left\{E_{\text {lowest }}(I), \frac{E_{\text {lowest }}(I+1)+E_{\text {lowest }}(I-1)}{2}\right\}$ in order to compare the four spectra. We then assign the label $N$ to the levels in the four spectra, counting from the lowest according to the excitation energy $E(I)-E_{r e f}(I)$ measured from the reference. By this definition the $N=1$ level represents the "strictly yrast" level in the sense that it refers to only one among the four lowest levels defined separately for the four parity and signature quantum numbers, while the other three are treated as excited levels $(N>1)$ with respect to the strict yrast level. Collecting the spacings from the $N$-th rotational band (they are the spacings between the $N$-th level and the next excited level with the same $I^{\pi}$ ) from the spin range $I_{0}=32-50$ in the 40 rare-earth nuclei, we calculate the NND and extract the Brody parameter for each $N$. We also made the same analysis for spin intervals, $I_{0}=20-30$ and $I_{0}=52-60$ in order to study a possible spin-dependence, although the present model may not be very realistic for the lower spin interval $\left(I_{0}=20-30\right)$ because of the problem of 


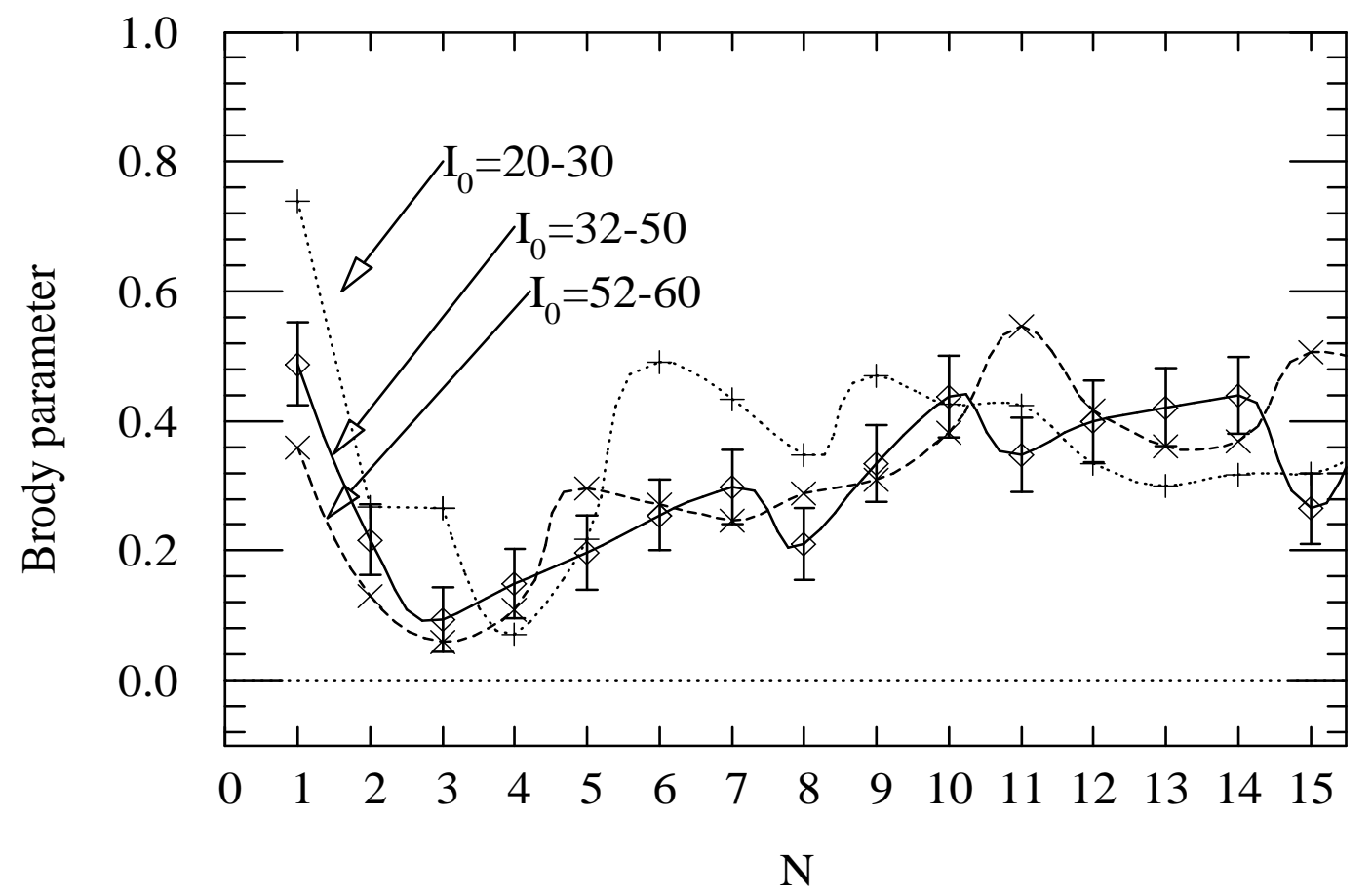

Figure 4: The Brody parameter extracted from the NND associated with the lowest 15 near-yrast states. See text for the definition of the strict ordering $N$ of the states. Different symbols represent spin intervals $I_{0}=32-50, I_{0}=20-30$, and $I_{0}=52-60$.

the pairing correlation.

The extracted Brody parameter is plotted in Fig. 4 as a function of the strict level order $N$, for the lowest 15 levels. The spacings analysed for Fig. 1 belong mostly within the first energy bin adopted in the previous subsection, which included the lowest 5 levels at each $I^{\pi}$. The Brody parameters plotted in Fig. A in average agree with the value $w \sim 0.3$ for the lowest bin in Fig. 1. It is seen in Fig. 4 that the Brody parameter gradually decreases as the levels become closer to the yrast line, except for $N=1$. For the lowest few levels, the Brody parameter is about 0.1-0.2, which is close to the Poisson limit (The corresponding NND's are shown in Fig. $5(b, c)$ for the third and tenth lowest levels $N=3$, and 10). This indicates that the excitation energy dependence shown in Fig.1. continues down to the lowest few states near the yrast line. However, a remarkable deviation from the overall excitation energy dependence is clearly noticed for the lowest point at $N=1$, i.e., for the spacings between the yrast band and the next excited band with same $I^{\pi}$, for which $w \approx 0.4-0.7$. The NND for $N=1$ is shown in Fig. 5 (a).

It is also seen that the spin dependence is not strong while at $N=1$ the Brody parameter at lower spins shows a more significant deviation from the Poisson limit, becoming close to the Wigner limit.

\subsection{Level spacing statistics at yrast}

In order to study the origin of the deviation of the first spacings from the Poisson distribution, we perform calculations neglecting the residual two-body interaction in which all the states become pure many-particle many-hole mean-field configurations. The NND and the extracted Brody parameter are compared in Figs. 5 and 6 with those obtained by inclusion of the residual interaction.

The Brody parameter of the lowest spacings $(N=1)$ is essentially unaffected by the inclusion of the residual interaction. This indicates that the deviation from the Poisson limit for $N=1$ does not originate from the residual interaction, but from the mean field. On the other hand, Figures 5 and 6 also show that for the higher spacings above $N \sim 3$, the Brody parameter of the pure mean 


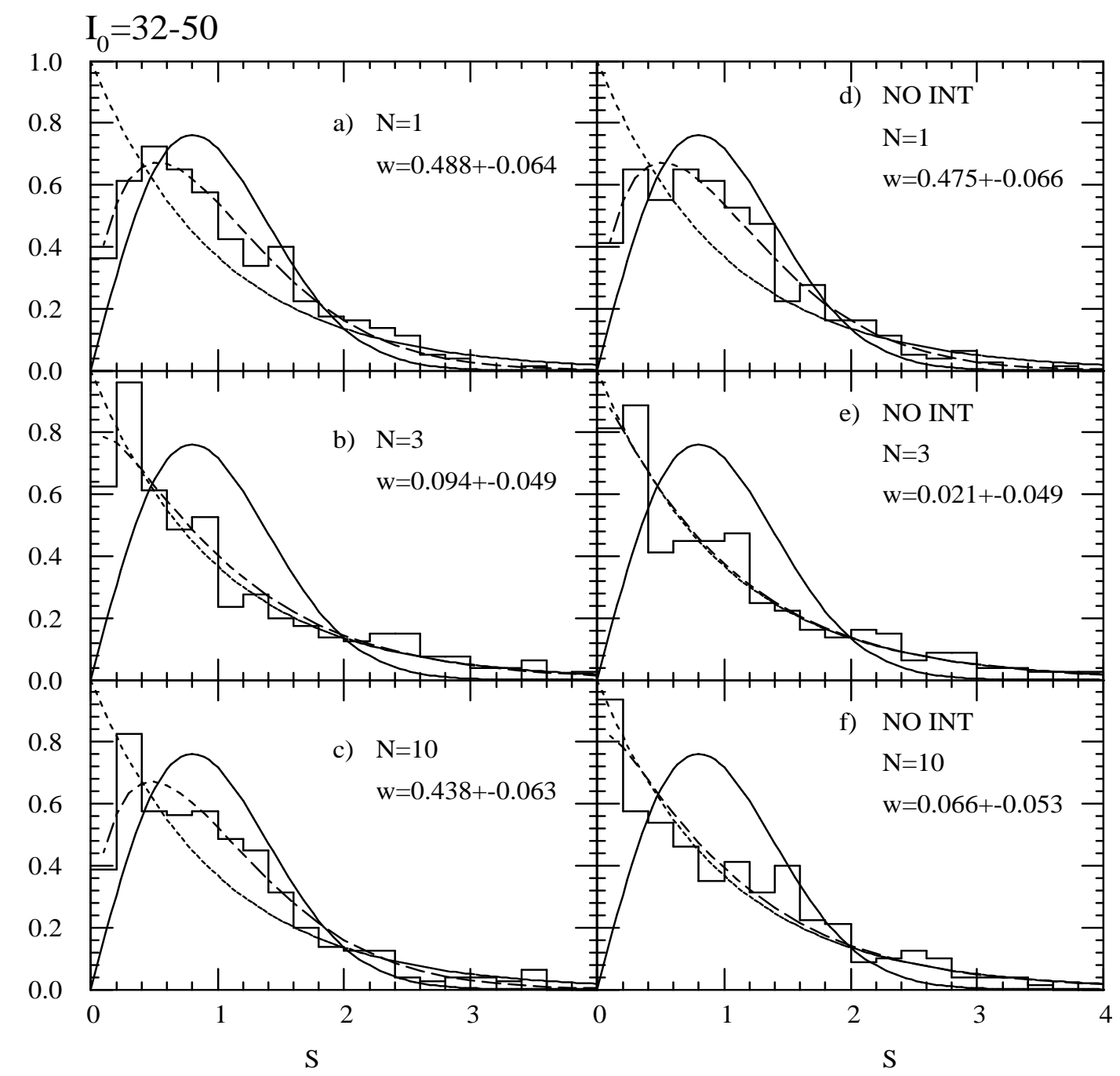

Figure 5: The NND associated with the lowest, third, and the tenth (strict order $N=1,3,10$ ) levels within the spin interval $I_{0}=32-50$ for (a), (b), and (c), respectively. (d,e,f) the same as (a,b,c) except that the residual SDI interaction is neglected. 


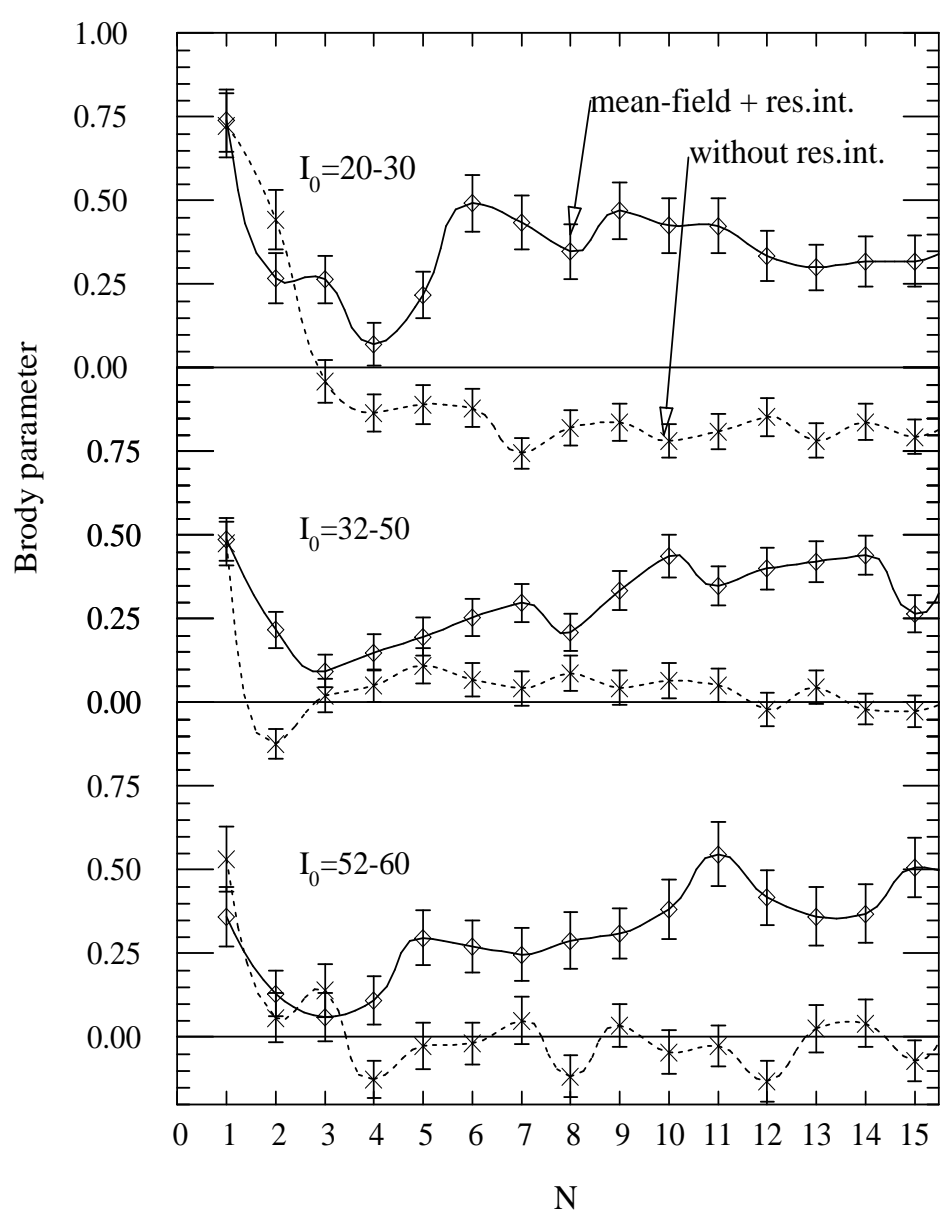

Figure 6: The Brody parameter extracted from the NND associated with the lowest 15 near-yrast states in the strict order obtained from the mean-field calculation without the residual interaction for different spin intervals $I_{0}=20-30, I_{0}=32-50$ and $I_{0}=52-60$ (points joined by dashed curves). It is compared with the result with the residual interaction (ones joined by solid curves. See also Fig.4). 
field calculation converges to the Poisson limit $w=0$ except for $I_{0}=20-30$ while the Brody parameter and the NND calculated with the residual two-body interaction deviates from the Poisson limit. This explicitly indicates that, contrary to the case of $N=1$, the deviation from the Poisson limit at $N \gtrsim 2$ arises from the residual two-body interaction.

We look for the origin of the special feature of the very yrast $N=1$ spacings in connection with the single-particle level structure in the cranked Nilsson mean-field. To this end, we first remark that for the levels near the yrast line, the configurations are only weakly mixed by the residual two-body force and most of them have essentially independent-particle configurations. In particular, the excited states near the yrast lines often have $1 \mathrm{p} 1 \mathrm{~h}$ configuration with respect to the yrast configuration. This means that the relatively large value of the Brody parameter extracted for the yrast levels may not be directly related to the mixture caused by the residual interaction. Furthermore, when the spin is not very large, the angular momentum alignments of intrinsic excitation is relatively small compared to the level spacings between the states in the yrast band and the next excited states with the same quantum number; it is found that the last term in Eq.(4) representing the alignment effect on the energy is at least a factor $\gtrsim 2$ smaller than the average level spacing $D \sim 350 \mathrm{keV}$ associated with the $N=1$ yrast rotational band. Under these conditions, the relative energy of the first excited states having the same $I^{\pi}$ measured from the yrast states can be approximated by the $1 \mathrm{p} 1 \mathrm{~h}$ excitation energy in the single-particle routhian spectrum. Namely, $E_{\text {next }}\left(I^{\pi}\right)-E_{\text {yrast }}\left(I^{\pi}\right) \sim$ $e_{p, \alpha \pi}^{\prime}\left(\omega_{I}\right)-e_{h, \alpha \pi}^{\prime}\left(\omega_{I}\right)$ where $e_{p, h}^{\prime}$ are the single-particle routhian of the involved particle and hole. The particle and hole orbits necessarily have the same quantum numbers (signature $\alpha$ and parity $\pi)$. On the other hand, spacings between excited states (yrare levels) do not keep such relation to the single particle spacings. This is illustrated in Fig. 7f which shows examples of the main mean field components of the lowest excitation within each parity-signature set of states. It is clearly seen how the excitation from the yrast state in this typical case will proceed by changing the orbit of one particle, keeping its $(\pi, \alpha)$ (as shown in the left panel of Fig.(7). For yrare levels, excitations relative to the Fermi surface are already present, and the lowest excitation starting from an yrare state most often will proceed by a $2 \mathrm{p}-2 \mathrm{~h}$ excitation which connect orbitals with different $(\pi, \alpha)$, as shown in the right panel.

In the previous section, we find that the the special feature seen for the yrast bands becomes more prominent as the spin decreases. This feature is consistent with the above interpretation. In addition, we find that there exists an odd-even effect for $N \lesssim 10$ for the low spins $I_{0}=20-30$; the level statistics for odd-odd nuclei is very close to the Poisson distribution while even-even or odd- $A$ nuclei shows significant deviation from the Poisson distribution for $N=1$. This also indicates that the level spacings associated with the very yrast states reflects the single-particle level spacings since the odd-even effects can arise from the fact that many cranked-Nilsson routhian orbits retains two-fold degeneracy (signature splitting is small) at low rotational frequency.

These considerations lead us to investigate the spacing distribution of the single-particle levels in the cranked Nilsson potential. Figure 8 shows distribution of the level spacings $e_{p, \alpha \pi}^{\prime}\left(\omega_{I}\right)-e_{h, \alpha \pi}^{\prime}\left(\omega_{I}\right)$ between the hole and particle orbitals having the same parity and signature quantum number which correspond to the lowest $1 \mathrm{p} 1 \mathrm{~h}$ excitations for all 40 nuclei and rotational frequencies corresponding to spin $I_{0}=30,32, \ldots 50$. We have not applied the unfolding procedure since the relevant single-particle orbits lie only in a limited region around the neutron and proton Fermi surfaces $(N=94-105, Z=$ $66-71$ ) of the cranked Nilsson spectrum. It is noticed in Fig.8 that the spacing distribution is concentrated around the average spacing $(\langle D\rangle \sim 400 \mathrm{keV})$ and there are few spacings smaller than $200 \mathrm{keV}$, indicating that degeneracy among orbits with the same quantum numbers happens only rarely. This in fact comes from the nature of the cranked Nilsson spectrum (which is believed to be valid also for other models such as Woods-Saxon potential). One of the relevant properties is that a large part of the deformed mean field is of harmonic oscillator type, and that the quantum spectrum of the oscillator shows strong level repulsion while the corresponding classical motion is integrable. With deformation not very large $(\epsilon \lesssim 0.3)$ the single-particle orbits around the Fermi

\footnotetext{
${ }^{1}$ For the rotational frequencies corresponding to the spin $I_{0}=20-30$, many of the cranked-Nilsson routhian orbits show very little signature splitting. This causes frequent near degeneracy in the $n \mathrm{p}-n \mathrm{~h}$ configurations, leading to the enhancement of the NND for small spacings and producing negative values of the Brody parameter for $N \gtrsim 3$ in $I_{0}=20-30$ case in Fig. 6 .
} 


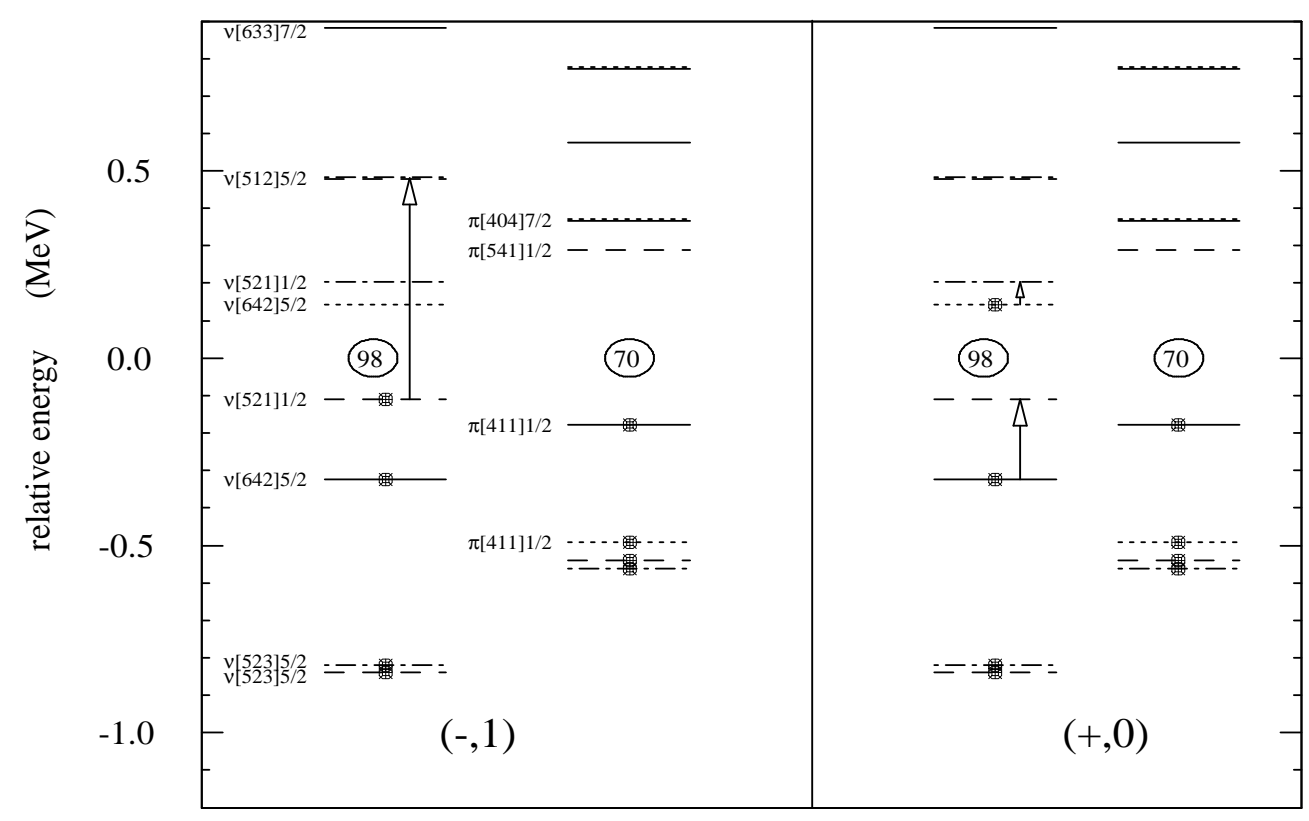

Figure 7: Main mean-field configurations in the lowest states with $(\pi, \alpha)=(-, 1)$ and $(+, 0)$ in ${ }^{168} \mathrm{Yb}$ at $\omega=0.319$ corresponding to $I=30,31$. The blobs represent the occupied orbitals in the main configuration of the lowest state for each $(\pi, \alpha)$. At this spin, the yrast state belongs to $(-, 1)$. The arrows represent excitations involved in the second lowest state in each $(\pi, \alpha)$. The solid,dotted, dashed and dot-dashed lines represent the cranked Nilsson single-particle orbits with $(\pi, \alpha)=(+, 1 / 2),(+,-1 / 2),(-, 1 / 2)$, and $(-,-1 / 2)$ respectively.

surface belong to a single major oscillator shell, provided that the parity and the kind of particle is fixed. Taking the neutron spectrum as an example, the negative parity orbits are dominated by those with the total oscillator quanta $N_{o s c}=5$. Because of the mean-field deformation, the orbits having different $n_{3}$ (oscillator quanta along the deformation axis) are then splitted in energy, and this makes degeneracy among orbits with different $n_{3}$ asymptotic number rare. Furthermore, the $l^{2}$ and $l s$ terms of the mean field cause splittings among the orbits having the same $n_{3}$. The positive parity neutron orbits near the Fermi surface are $i_{13 / 2}$ orbits, and because of the deformation splitting, the $i_{13 / 2}$ orbits with fixed signature are placed with finite intervals at any rotational frequency. Therefore degeneracy among the $i_{13 / 2}$ orbits never happens. An additional mechanism arises from the fact that the nuclear mean-field favours an equilibrium deformation at which the shell energy lowers, implying that degeneracy of single-particle orbits at the Fermi surface is unfavoured. All these mechanisms prefer to the Wigner-like distribution in the single-particle spacing at the Fermi surface. Consequently, there exist only few cases of small spacings as seen in Fig.8, especially for the low spin region $I_{0}<30$. At higher spins (i.e., at high rotational frequency), some specific orbits with large rotational alignment, e.g, proton orbits stemming from $h_{9 / 2}$ and $i_{13 / 2}$ intrudes in the Fermi surface region around $I \sim 40-50$ and cross sharply with other orbits. Small spacings associated with these highly aligned orbits are present in the distributions shown in Fig. \&(b) for $I_{0}=32-50$ (and slightly also for $I_{0}=52-60$ ), but this does not enhance very much the probability of small spacings and keep the distribution Wigner-like.

Consequently, the intrinsic nature of the cranked single-particle spectrum affects specifically the level spacing distribution associated with the yrast band at $N=1$. Figure 6 indicates that this remains to some extent even for the very high spin region with $I=30-60$. By the same token, the present analysis suggests that the singular behaviour of the lowest spacing could be stronger at lower spins. It should be remarked however that the present model is not very accurate for describing the near-yrast rotational bands at lower spins since the pairing correlation which is important at low spins is not well taken into account. Thus, the results of the present analysis cannot readily be compared to experiments for the lower spins $(I \lesssim 30)$. 

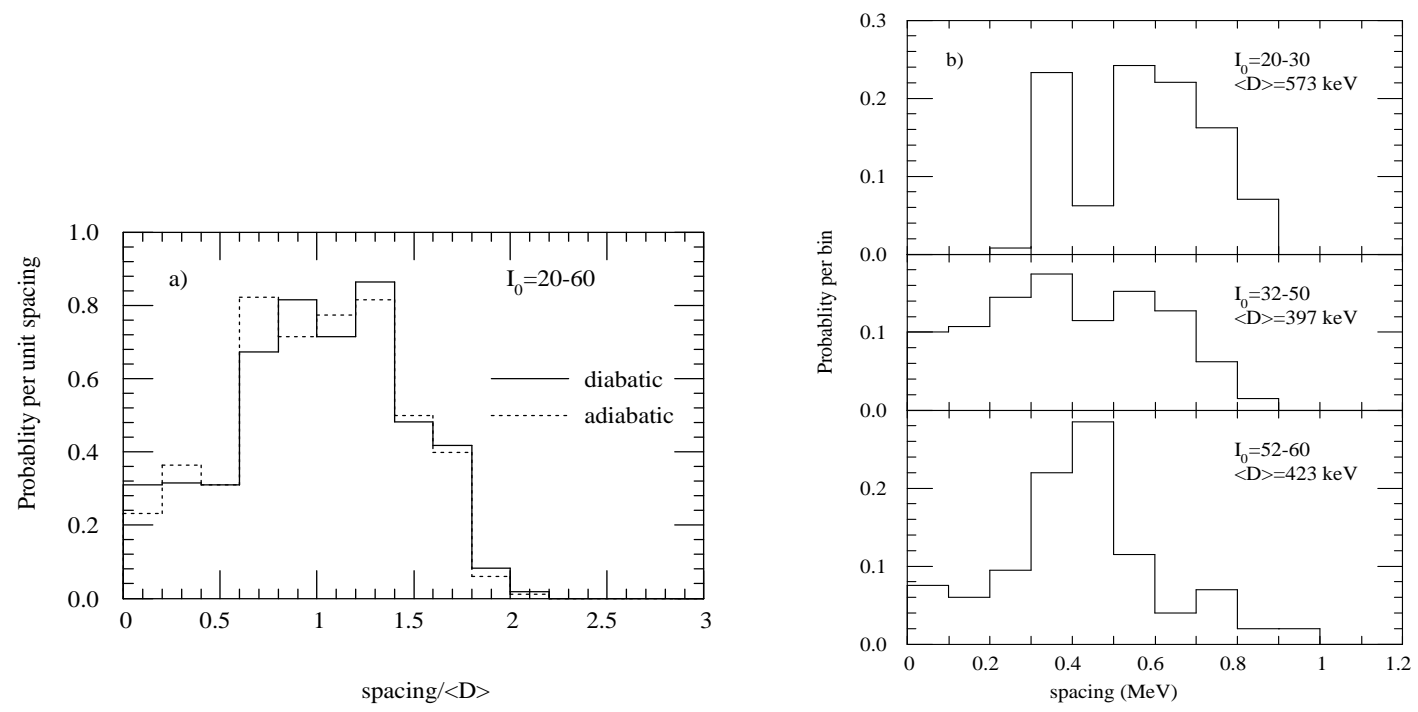

Figure 8: (a) The distribution of the spacings of the cranked Nilsson single-particle orbits for spin interval $I_{0}=20-60$. The sampling is described in the text. The dotted line represents the distribution which is obtained when the adiabatic basis is adopted. There is no significant difference between the diabatic and adiabatic basis. (b) The same as (a), but the histogram bins are defined with the spacing itself instead of the normalized spacing, and also the spin interval is subdivided into $I_{0}=20-30, I_{0}=32-50$ and $I_{0}=52-60$.

We have here related the angular momentum dependence of the spacings of the single-particle spectrum in the mean field, displayed in Fig.8(b), to the behavior of specific important orbits in the cranked potential. One may ask whether a more consistent explanation may be achieved from the general properties of the phase space associated with the classical single-particle motion in the rotating potential. So far, the questions of chaotic and regular motion in rotating deformed potentials have only been carried out for billiards in two dimensions 42, 43. It is found 42] that rotation certainly may affect the phase space generally. However, especially for the high kinetic energies of nucleon states around the Fermi surface, the predicted effects are small.

The absolute nature of the yrast band relative to yrare bands of the other parity-signature configurations is worth emphasizing. We have discussed it from our model, especially by means of Fig.7. However, it may actually be less specific to the actual model, since it could result from a general homo-lumo gap in a quantal system, but now for states within an interval of angular momenta. The favoured yrast configuration should then be able to determine the detailed shape and other properties of the nucleus, and the yrare levels then have to adjust to this.

\subsection{Analysis without unfolding procedure}

As described in Sect.2, we apply the unfolding procedure in order to separate the overall excitation energy dependence and the local fluctuations in the level spacings. This procedure, however, cannot be used for the analysis of the present experimental data in the high spin region. In fact, the number of identified levels at fixed $I^{\pi}$ is far below 10 at high spins in the experiments performed so far. The experimental analysis in Refs. 11, 12, 13 does not apply such an unfolding procedure that is described in Sect.2. In order to facilitate a direct comparison between the theoretical results and experiments, we propose in this subsection another way of analysis which does not use the unfolding procedure, but still takes into account the excitation energy dependence of the level density in an approximate way. The procedure is also applicable to the analysis of experimental data.

Although the level density increases exponentially with increasing intrinsic excitation energy $U$, it may be assumed that the level density at given $U$ is rather independent of spin and parity and nuclear species as far as the high spin states in the same mass region are concerned. In fact, as 


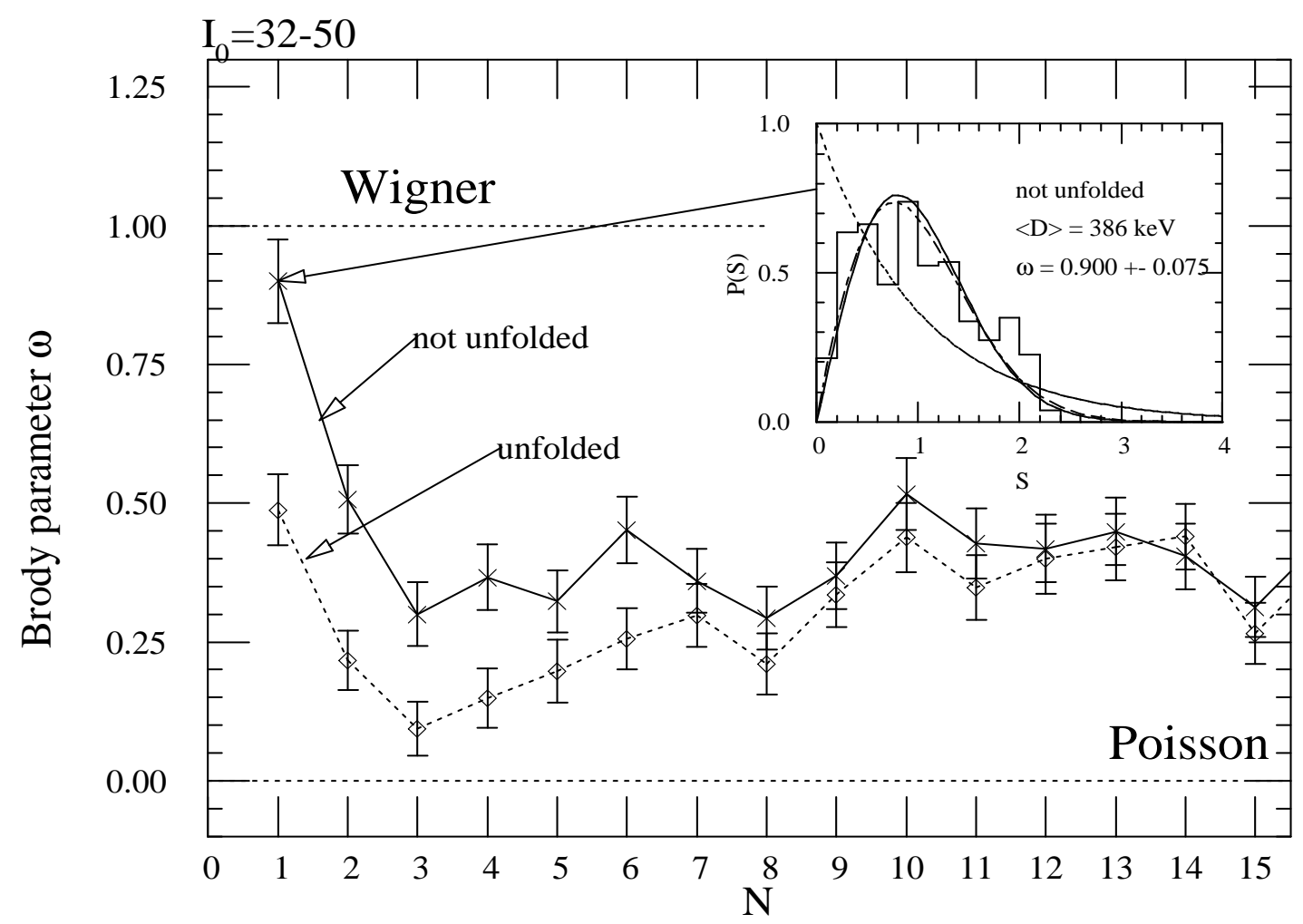

Figure 9: The Brody parameter extracted from the NND obtained by using the simple normalization without the unfolding procedure for the spin interval $I_{0}=32-50$ as a function of the strict level ordering $N$ (the symbols connected with the solid line), compared with that obtained with the unfolding (dotted line). The inset shows the NND for $N=1$ obtained without unfolding.

discussed in Ref. [44], the level density at fixed $I^{\pi}$ can be accounted by the level density of intrinsic configurations in the cranking model if the spin is sufficiently high (e.g. above $I \gtrsim 12$ for $U<3 \mathrm{MeV}$ ). In this limit, the level density may be approximated by the Fermi gas formula [44] as a function of a single variable $a U$ where $a$ is the level density parameter related to the single-particle level density at the Fermi surface of the cranked mean-field.

Keeping this in mind, let us consider an ensemble of level spacings which is specified by the strict level ordering $N$ as introduced in the previous subsections. The level spacings within this ensemble are expected to have a common average value since the level ordering $N$ and hence the excitation energy is taken to be the same. It then may be reasonable to define, without using the unfolding procedure, the normalized spacing $s=D /\langle D\rangle$ by simply dividing the spacing $D$ by the average spacing $\langle D\rangle$ calculated for this ensemble specified by $N$. We show in Fig.9 the NND and the extracted Brody parameter calculated in this way. It is seen that there exits small but systematic difference for $N \lesssim 5$ between the results calculated with and without the unfolding procedure. The origin of the difference can be understood by noting that the average level spacings for the lowest states are $386,271,214, . .163 \mathrm{keV}$ for $N=1,2,3, . .5$, which are not very small compared with the temperature parameter $T \sim 350 \mathrm{keV}$ in the fitted level density. In other words, the smooth level density $\bar{\rho}(E)$ varies significantly in the energy interval of the single spacing, especially if the lowest few $N$ 's are concerned. This causes a difference in the profile of the NND depending on whether we adopt the unfolding procedure or not. However, it should be stressed that, in spite of the difference depending on the way of analysis, the Wigner-like property associated with the yrast spacings $(N=1)$ is present in both analysis. It becomes even more significant with the simple way of analysis without the unfolding procedure.

Except for the lowest several spacings in the strict ordering, the NND obtained by means of the simple normalization agrees with those obtained with the unfolding procedure. As another 


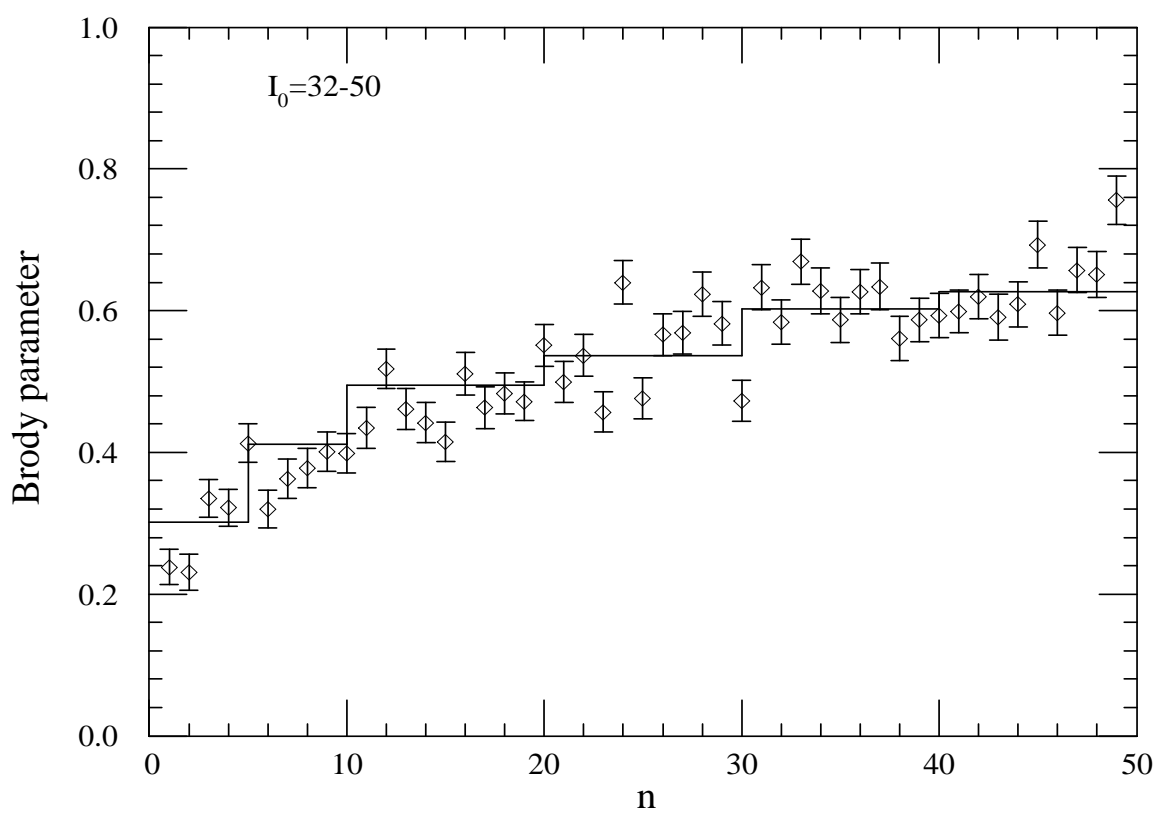

Figure 10: The Brody parameter extracted from the NND associated with the $n$-th level at each $I^{\pi}$ for spin interval $I_{0}=32-50$ (See text). Here the unfolding procedure is not applied. The result is compared with the one with the unfolding procedure which is calculated for the bins of levels (same as the solid line in Fig.1).

illustration, we consider an ensemble of level spacings specified by the level ordering $n$ defined in each spectrum for each $I^{\pi}$ (note the difference between $n$ and the strict ordering $N$ ), and calculate the NND and the Brody parameter for spin interval $I_{0}=32-50$ by means of the simple normalization procedure described above. In this case, the average spacing $\langle D\rangle$ is calculated for each $n$. The result is compared in Fig.10 with the Brody parameter (Fig.1 in subsect.3.1) analysed for level bins $n=1-5,5-10,11-20, \ldots$ by using the unfolding procedure. Both ways of analysis agree very well with each other, leading to the same conclusion about the overall excitation energy dependence of the NND. The NND's for $n=1$ and 2 are plotted in Fig.11. $(\mathrm{a}, \mathrm{b})$.

\subsection{Relation to experimental analysis}

The experimental NND obtained by Shriner et al. [8] for the low-lying low-spin states $(I \lesssim 5 \hbar)$ in rare-earth nuclei displays a Brody parameter around 0.3. A more recent analysis which includes the observed rotational states at relatively high spins (most of the analysed levels have $I \lesssim 30$ ) reports a NND which is close to the Poisson distribution [11, 12, 13]. Our theoretical calculations for higher spins $I \gtrsim 30$ also favours a Poisson-like NND for the levels near the yrast line. In the following we try to perform our theoretical analysis in a way similar to the procedure adopted by Garrett et al. [11, 12, 13. One should however remark that a comparison between our results and the experimental findings can only be indicative, because they refer to different spin regions.

In accordance with Ref.[11], we consider here an ensemble of the spacings associated with the lowest and second lowest states at each $I^{\pi}(n=1$ and 2). However we deal with the spin interval $I_{0}=32-50$, where the pairing effects are expected to be weak. We remark that the pairing effects are removed to some extent from the experimental analysis by excluding the lowest $(0,+)$ spacings [11. We adopt the normalization scheme introduced in Subsect.3.4 which do not use the unfolding procedure since the experimental analysis 11] adopt the similar normalization. The obtained NND's, shown in Fig.11 (a,b), are close to the Poisson distribution, having Brody parameter $w \sim 0.25$ in agreement with the Poisson-like NND seen already for the near-yrast states. It also shares some common features with the experimental analysis [11]: A deviation from the Poisson distribution is seen for small spacings $s \lesssim 0.2$ and is most significant for the smallest spacing with $s \lesssim 0.1$ or $D \lesssim 25$ 


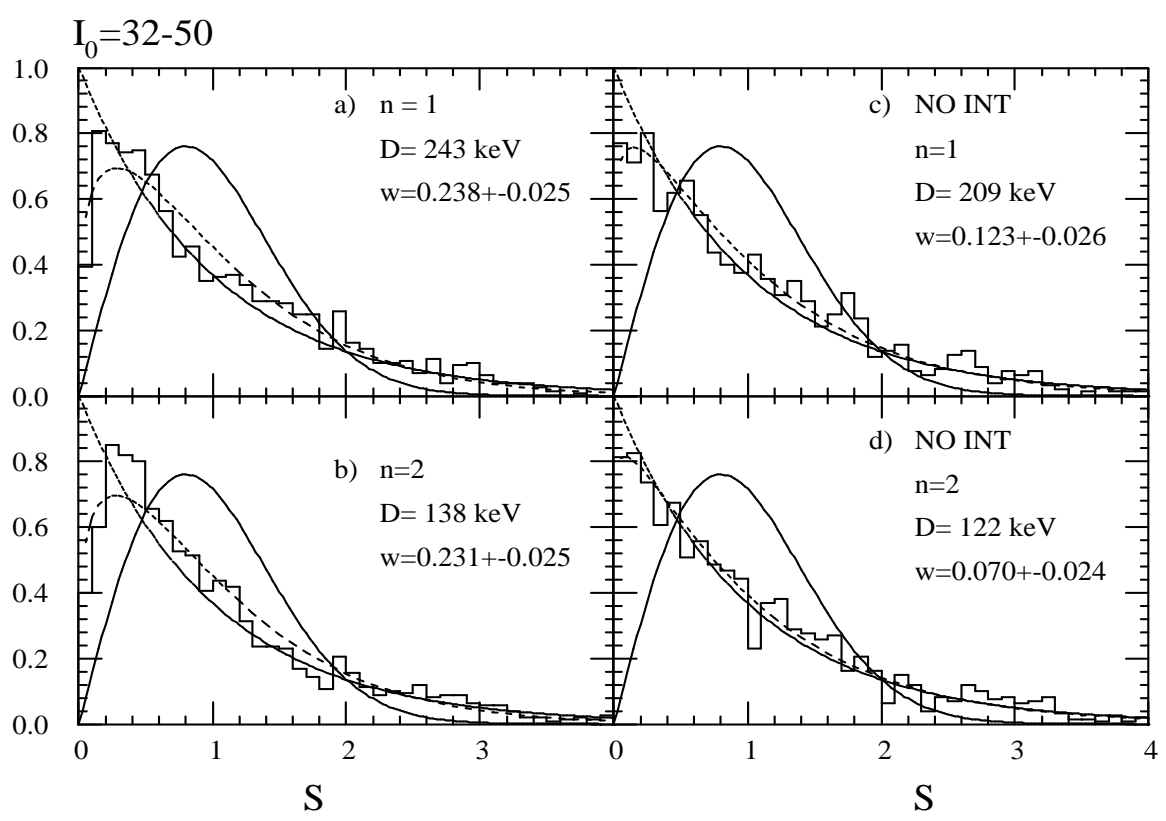

Figure 11: The Brody parameter extracted from the NND associated with the lowest and second lowest states $(n=1,2)$ at every $I^{\pi}$, for (a) and (b), respectively. Here the unfolding procedure is not applied, and the spacings are normalized to the average spacing defined in each ensemble. (c) and (d), the same as (a) and (b) except that the residual SDI interaction is neglected and pure mean-field many-particle many-hole configurations are considered.

$\mathrm{keV}$.

In Fig.11(c,d), we also calculated the NND in the same way except that the residual two-body interaction is neglected. Comparing Fig.11 (a,b) and Fig.11(c,d), it is indicated that the deviation from the Poisson limit at small spacings, seen in Fig.11 (a,b), is mostly caused by the residual twobody interaction. We remark, however, that the Wigner-like distribution associated with the very lowest spacing $N=1$ discussed in Subsect. 3.3 should be present, but is not visible neither in Fig.11.(a) nor in (c). This is because the ensemble with $n=1$ contains also the other spacings with $N=2,3, .$. , which have lower average spacing and mask the Wigner-like distribution associated with $N=1$ spacings. This suggests that, in order to find the Wigner-like distribution caused by the mean-field effect in the experimental analysis, one should make an analysis by subdividing the ensemble of the spacings with respect to the strict level order $N$.

\section{Conclusions}

We analysed the level statistics of the high spin states with $I \gtrsim 30$ in rare-earth deformed nuclei as a function of the intrinsic excitation energy of the rotating nuclei. We used a shell model approach which describes $n \mathrm{p}-n \mathrm{~h}$ excitations in the cranked Nilsson potential interacting through the surfacedelta residual interaction. We put emphasis on the analysis of the near-yrast levels which may be accessible by discrete $\gamma$-ray spectroscopies.

The nearest neighbour level spacing distribution (NND) and the $\Delta_{3}$ statistics indicate that the level fluctuations in the near-yrast region follow a distribution close to the Poisson limits with the extracted Brody parameter $w=0.2-0.3$. This value of the Brody parameter implies significant deviation from the Poisson distribution for spacing smaller than about $s \lesssim 0.3$. The experimental analysis of the NND for low-spin states [8] and the one including high spin rotational states [11, 12, 13] indicates a Poisson-like distribution in the near-yrast states. The present analysis suggests that this behaviour extends up to very high spins $I \sim 50-60$. The level statistics approaches the GOE limit as the intrinsic excitation energy $U$ increases, but this process proceeds very gradually and the chaos 
limit is nearly attained only with $U \gtrsim 2 \mathrm{MeV}$. This transition is caused by the residual two-body interaction.

An interesting aspect of the NND emerges when we focus on the lowest levels near the yrast line. The level spacings between the yrast rotational band and the next excited band with the same spin and signature favour a Wigner-like NND, rather than obey the Poisson-like distribution associated with the other near-yrast levels. The distinguishable property of the NND associated with the yrast rotational bands arises from the mean-field property of the rotating nuclei while the deviation from the Poisson limit seen for the other spacings among yrare rotational bands is caused by the residual two-body interaction. Since the lowest few levels near yrast have dominantly one particle excitations, the spacing between the yrast rotational level and the next excited level thus reflects the single-particle routhian spectrum in the rotating mean-field at equilibrium normal deformation, which shows a Wigner-like distribution for the spacings between orbits with the same parity and signature around the Fermi surface.

\section{Acknowledgments}

Discussion with J.D. Garrett is acknowledged. One of the author, M.M., thanks the Danish Research Council for support of his stay at Niels Bohr Institute where a part of the research reported here was carried out.

\section{References}

[1] T.A.Brody, J.Flores, J.B.French, P.A.Mello, A.Pandy and S.S.M.Wong, Rev. Mod. Phys. 53(1981) 385.

[2] C.E. Porter, Statistical theories of spectra: Fluctuations (Academic Press, 1965).

[3] M.L. Mehta, Random matrices and the statistical theory of energy levels (Academic Press, 1967).

[4] R.U.Haq, A.Pandey, and O.Bohigas, Phys. Rev. Lett. 48 (1982) 1086.

[5] O. Bohigas, M.J. Giannoni and C. Schmit, Phys. Rev. Lett. 52(1984) 1.

[6] O. Bohigas and H.A. Weidenmüller, Ann. Rev. Nucl. Part. Sci. 38 (1988) 421.

[7] A.Y. Abul-Magd and H.A. Weidenmüller, Phys. Lett. B162 (1985) 223.

[8] J.F.Shriner,Jr., G.E.Mitchell, and T. von Egidy, Z. Phys. A338(1991) 309.

[9] J.F.Shriner,Jr., E.G.Bilpuch, P.M.Endt and G.E.Mitchell, Z. Phys. A335(1991) 393.

[10] S. Raman, T. A. Walkiewicz, S. Kahane, E. T. Jurney, J. Sa, Z. Gacsi, J. L. Weil, K. Allaart, G. Bonsignori, and J. F. Shriner,Jr., Phys. Rev. C43 (1991) 521.

[11] J.D. Garrett, J.Q. Robinson, A.J.Foglia, and H.-Q.Jin, preprint 1996.

[12] J.D. Garrett, J.R. German, L. Courtney, and J.M. Espino, Proc. Symp. on Future Directions in Nuclear Physics with $4 \pi$ Gamma Detection Systems of the New Generation, Strasbourg, 1991, eds. J. Dudek and B. Haas (American Institute of Physics, 1992) p.345.

[13] J.D. Garrett, Proc. the Eighth Int. Symp. on Capture Gamma-Ray Spectroscopy and Related Topics, Fribourg, 1994, ed. J. Kern (World Scientific, 1994). 
[14] R. Bengtsson and S. Frauendorf, Nucl. Phys. A314(1979) 27; A327(1979) 137.

[15] T.Bengtsson and I.Ragnarsson, Nucl. Phys. A436(1985) 14.

[16] As reviews, S.Åberg, H.Flocard, W.Nazarewicz, Ann. Rev. Nucl. Part. Sci, 40 (1990) 439; Z.Szymanski, Fast Nuclear Rotation (Clarendon Press, Oxford, 1983);

M.J.A. de-Voigt, J.Dudek, Z.Szymansky, Rev. Mod. Phys. 55 (1983) 949.

[17] B.Lauritzen, T.Døssing and R.A.Broglia, Nucl. Phys. A457(1986) 61.

[18] B.Herskind, A.Bracco, R.A.Broglia, T.Døssing, A.Ikeda, S.Leoni, J.Lisle, M.Matsuo, and E.Vigezzi, Phys. Rev. Lett. 68(1992) 3008;

T. Døssing, B. Herskind, S. Leoni, M. Matsuo, A. Bracco, R.A. Broglia, and Vigezzi, Phys. Report 268 (1996) 1.

[19] S.Åberg, Phys. Rev. Lett. 64(1990) 3119;

S.Åberg, Prog. Part. Nucl. Phys. vol.28 (Pergamon 1992) p.11.

[20] M. Matsuo, T. Døssing, E. Vigezzi, R.A. Broglia, and K. Yoshida, preprint YITP-96-48 1996, Nucl. Phys. in press.

[21] I. M. Green and S. A. Mozkowski, Phys. Rev. 139 (1965)B790;

R.Arvieu and S.A.Mozkowski,Phys. Rev. 145 (1966) 830.

[22] A. Faessler, Fortschr. Phys. 16(1968) 309.

[23] M. Matsuo, T. Døssing, E. Vigezzi and R.A. Broglia, Phys. Rev. Lett. 70 (1993) 2694;

M. Matsuo, T. Døssing, B. Herskind, S. Frauendorf, E. Vigezzi and R.A. Broglia, Nucl. Phys. A 557 (1993) 211c.

[24] A. Bracco, P. Bosetti, S. Frattini, E. Vigezzi, S. Leoni, T. Døssing, B. Herskind, and M. Matsuo, Phys. Rev. Lett. 76 (1996) 4484.

[25] T.Mizusaki, T.Otsuka, and P.von Brentano, Nucl. Phys. A598 (1996) 47.

[26] A.Alhassid and D.Vretenar, Phys. Rev. C46 (1992) 1334.

[27] A.T.Kruppa, K.F.Pal, N.Rowley, Phys. Rev. C52 (1995)1818.

[28] J.D.Garrett, Nuclear Structure 1985, eds. R. Broglia, G. B. Gagemann and B. Herskind (Elsevier Science,1985) p.111.

[29] Y.R.Shimizu, J.D.Garrett, R.A.Broglia, M.Gallardo and E.Vigezzi, Rev. Mod. Phys. 61 (1989) 131.

[30] Y.R.Shimizu, Nucl.Phys., A520 (1990) 477c.

[31] S. Åberg, Phys. Scr. 25 (1982) 23.

[32] T.R. Werner and J. Dudek, Atomic Data and Nuclear Data Tables, 50(1992) 179.

[33] R.Bengtsson, S.Frauendorf and F.-R.May, Atomic Data and Nuclear Data Tables, 35(1986) 15.

[34] O. Bohigas and M.J. Giannoni, Mathematical and Computational Methods in Nuclear Physics, Lecture Notes in Physics 209, eds. J.S.Dehesa, J.M.G. Gomez and A.Polls (Spriger 1984) p.1.

[35] A.Gilbert and A.G.W.Cameron, Can. J. Phys. 43 (1965) 1446.

[36] F.J. Dyson and M.L. Mehta, J. Math. Phys. 4 (1963) 701.

[37] P.Persson and S. Åberg, Phys. Rev E52 (1995) 148.

[38] M.V. Berry, Proc. R. Soc. Lond., A400(1985) 229. 
[39] P. Arve, Phys. Rev. A44(1991) 6920.

[40] A. Fitzpatrick, S.A. Araddad, R. Chapman, J. Copnell, F. Lind'en, J.C. Lisle, A.G. Smith, J.P. Sweeney, D.M. Thompson, W. Urban and S.J. Warburton, J. Simpson, C.W. Beausang, J.F. Sharpey-Shafer, S.J. Freeman, S. Leoni, and J. Wrzesinski,

Nucl. Phys. A585 (1995)335.

[41] A. Nordlund, R. Bengtsson, P. Ekström, M. Bergström, A. Brockstedt, H. Carlsson, H. Ryde, Y. Sun, A. Atac, G.B. Hagemann, B. Herskind, H.J. Jensen, J. Jongman, S. Leoni, A. Maj, J. Nyberg and P.O. Tjøm, Nucl. Phys. A591(1995)117.

[42] H. Frisk and R. Arvieu, J. Phys. A22 (1989) 1765;

H. Frisk and R. Arvieu, Nucl. Phys. A495 (1989) 291c.

[43] A.J.S. Traiber, A.J. Fenrdik, and M. Bernath, J. Phys. A23 (1990) L305.

[44] S.Åberg, Nucl. Phys. 477 (1988) 18. 